\title{
Görland, Stephan O.: Medien, Zeit und Beschleunigung. Mobile Mediennutzung in Interimszeiten
}

\author{
Wiesbaden: Springer VS 2020. 235 Seiten. Preis: $€ 34,99$ (e-book)
}

\author{
Stephan Niemand
}

Angenommen: 9. Dezember 2020 / Online publiziert: 18. Dezember 2020

(C) Der/die Autor(en) 2020

Stephan Görland widmet sich in seiner Dissertation den Zeitpunkten im Tagesverlauf, die vor Verbreitung mobiler Medien überwiegend als ,unproduktive ' oder gar ,verlorene" Zeit wahrgenommen wurden: dem Warten in einer Arztpraxis, dem Fahren mit dem Zug oder dem Toilettengang. Diese Interimszeiten - so seine Begriffsbestimmung - können im ,digitalen Zeitalter' durch die Nutzung neuer mobiler Medien wie dem Smartphone auf vielfältige Art und Weise sinnstiftend gefüllt werden. Ob aus diesen neuen Nutzungsmustern ein Gefühl der subjektiven Beschleunigung erfolgt, ist ein zentrales Erkenntnisinteresse dieser Arbeit. Konkret fragt sich Stephan Görland: „Inwieweit besteht ein Zusammenhang zwischen Zeiterleben und der Nutzung mobiler Medien in Warte- und Transitzeiten?“ (S. 5). Seine Rezeptionsstudie, die im Kontext des DFG-Projekts „Mediated Time“ entstanden ist, zielt somit auf makroorientierte Diagnosen einer Beschleunigung des gesellschaftlichen Lebens, die auf der Mikroebene im Zeiterleben der mobilen Mediennutzung sichtbar werden müssten.

Schlüssig strukturiert er den Theorierahmen in drei Kapitel und exploriert so instruktiv seinen Forschungsgegenstand. Zunächst erfolgt in Kap. 2 eine differenzierte Betrachtung des (keineswegs neuen) Phänomens mobiler Mediennutzung. Überzeugend entfaltet er hier seine Arbeitsdefinition: Mobile Medien werden ,als portable und multimediale Geräte aufgefasst, die es permanent ermöglichen, in verschiedenen Kontexten wechselseitige Kommunikation zu betreiben.“ (S. 19). Angelehnt an diese Definition schließt er tragbare Medien wie E-Book-Reader oder nicht-internetfähige Handys von seiner Untersuchung aus und fokussiert sich folgerichtig auf das Smartphone. Kap. 3 widmet sich der komplexen Beziehung zwischen Me-

\footnotetext{
Dr. S. Niemand $(\bowtie)$

Institut für Kommunikationswissenschaft, Westfälische Wilhelms-Universität Münster, Bispinghof 9-14, 48143 Münster, Deutschland

E-Mail: stephan.niemand@uni-muenster.de
} 
dien(technologien) einerseits und (sozial konstruierten) Zeitordnungen sowie dem individuellen Zeithandeln im Alltag andererseits. Eindrücklich wird im Besonderen das Phänomen der permanenten Konnektivität erörtert, das sich im Zuge der Verbreitung des Smartphones als „das neue und allumfassende Strukturmerkmal“ (S. 32) herauskristallisiert und mit spezifischen Dynamiken innerhalb verschiedener Zeitpraktiken im Medienalltag verbunden ist. So zum Beispiel mit einer Fragmentierung in Form der Zersplitterung bestimmter Medienaktivitäten an verschiedenen Orten und zu verschiedenen Zeiten (wie die Rezeption einzelner Beiträge der Tagesschau über den Tag verteilt), mit einer Vergleichzeitigung der Nutzung verschiedener Mediendienste (wie die nahezu gleichzeitige Kommunikation mit verschiedenen Personen über Soziale Medien und E-Mail während der Fernsehrezeption) oder mit einer Habitualisierung (wie das kontinuierliche ,Checken“ des Smartphones). Diese neuen Formen des Zeithandelns mit (digitalen) Medien werden in Kap. 4 vor dem Hintergrund der populären Zeitdiagnose einer gesamtgesellschaftlichen Beschleunigung aufschlussreich und auch kritisch-reflexiv diskutiert. Der Wandel der Medienumgebung - etwa in Form der rasanten Verbreitung des Smartphones - lässt sich somit als ein Moment von Beschleunigung deuten, das im Medienhandeln in Gefühlswahrnehmungen wie Zeitdruck, einer diffusen Angst etwas zu verpassen oder neuen Formen des Erreichbarkeitsdrucks sichtbar werden kann. Gleichzeitig betont Stephan Görland, dass in solchen Diagnosen die sinnstiftende Komponente der Nutzung von (mobilen) Medien nicht aus dem Blick geraten darf. So wird in Kap. 5 - in dem auch die fundierte Begriffsbestimmung von Interimszeiten erfolgt - aus dem Forschungsstand zur Mediennutzung in Warte- und Transitzeiten abgeleitet, dass gerade Medien solche zeitlichen Abschnitte aufwerten können, indem beispielsweise Langeweile vorgebeugt oder Zeit effizient gestaltet wird.

Für die empirische Analyse, inwieweit sich mit dem Smartphone spezifische Nutzungsmuster in Interimszeiten herausbilden und wie sich dies auf die Zeitwahrnehmung und Zeitpraktiken auswirkt, wählt er eine Methodentriangulation aus einer InSitu-Befragung (ISS), einer Befragung als Experience Sampling Method (ESM) sowie qualitativen Leitfadeninterviews. Die Auswahl der konkreten Erhebungsinstrumente ist sinnvoll abgestimmt und stets schlüssig in Rückbezug zu den spezifischen Herausforderungen der Operationalisierung begründet. Der Einsatz der verschiedenen Instrumente erfolgte schrittweise (ISS 2015, ESM 2016, Interview 2017). Etwas überraschend wurde für jedes Erhebungsverfahren ein separates Sample gebildet. Zwar konnten die Instrumente so in Anlehnung an die vorherige Erhebung jeweils optimiert werden, ein möglicherweise erhellendes In-Beziehung-Setzen der verschiedenen Datensätze, wie es bei der Befragung der gleichen Personen möglich gewesen wäre, musste aber entfallen.

Gleichwohl werden mittels dieses aufwendigen Methodensettings eindrückliche und vielschichtige Befunde zum Erkenntnisinteresse generiert, deren Lektüre im Einzelnen empfehlenswert ist. An dieser Stelle seien zwei Einsichten herausgestellt: Erstens zeigt sich, dass eine gefühlte Beschleunigung in Interimszeiten mit einer Fragmentierung, Vergleichzeitigung und Habitualisierung der Smartphonenutzung verbunden ist. All diese Zeitpraktiken führen dazu, dass Interimszeiten ,mit einem mehr an Aktivitäten gefüllt bzw. verdichtet sind“ (S. 175) und als beschleunigt wahrgenommen werden. Indem zweitens neben den Interimszeiten auch Mediennutzung 
und Zeiterleben in weiteren Kontexten wie dem Zuhause oder im öffentlichen Raum erfasst wurden, ließ sich aufzeigen, dass - gefolgt von der Sphäre der Arbeit Interimszeiten am stärksten von einer gefühlten Beschleunigung betroffen sind. Im Kontext der Verbreitung des Smartphones, so ein zentrales Fazit, scheint das heutige Zeiterleben ,anders strukturiert [zu sein] als zu früheren Zeiten.“ (S. 184).

Abschließend lässt sich resümieren, dass sich die mit Mediatisierungsprozessen verwobene Beschleunigung des gesellschaftlichen Lebens gewiss nicht vollends über die Analyse der Smartphonenutzung in Interimszeiten untersuchen lässt und sicherlich wäre eine Analyse instruktiv, die den Wandel des Zeiterlebens über einen längeren Zeitraum zu verschiedenen Zeitpunkten erfasst, um die Veränderungen empirisch präzise nachzeichnen zu können. Aber: Stephan Görland legt mit seiner Dissertation $\mathrm{zu}$ diesem Themenfeld eine äußerst originelle und theoretisch fundierte Arbeit vor, die in der Argumentation stets überzeugen kann. Mit seinen Befunden aktualisiert er kommunikationswissenschaftliche Einsichten zur eher vernachlässigten Beziehung zwischen Zeiterleben und Mediennutzung. Zudem schafft er mit seiner Studie die Basis, um zukünftig (z. B. im Rahmen einer historisch orientierten Forschungsperspektive) tiefgründige Aussagen über den Wandel dieses Zusammenhangs treffen zu können. Kurzum: Wer sich für den Konnex Medien - Zeit - Beschleunigung interessiert, kommt an dieser Arbeit nicht vorbei.

Funding Open Access funding enabled and organized by Projekt DEAL.

Open Access Dieser Artikel wird unter der Creative Commons Namensnennung 4.0 International Lizenz veröffentlicht, welche die Nutzung, Vervielfältigung, Bearbeitung, Verbreitung und Wiedergabe in jeglichem Medium und Format erlaubt, sofern Sie den/die ursprünglichen Autor(en) und die Quelle ordnungsgemäß nennen, einen Link zur Creative Commons Lizenz beifügen und angeben, ob Änderungen vorgenommen wurden.

Die in diesem Artikel enthaltenen Bilder und sonstiges Drittmaterial unterliegen ebenfalls der genannten Creative Commons Lizenz, sofern sich aus der Abbildungslegende nichts anderes ergibt. Sofern das betreffende Material nicht unter der genannten Creative Commons Lizenz steht und die betreffende Handlung nicht nach gesetzlichen Vorschriften erlaubt ist, ist für die oben aufgeführten Weiterverwendungen des Materials die Einwilligung des jeweiligen Rechteinhabers einzuholen.

Weitere Details zur Lizenz entnehmen Sie bitte der Lizenzinformation auf http://creativecommons.org/ licenses/by/4.0/deed.de.

Dr. Stephan Niemand ist wissenschaftlicher Mitarbeiter im Arbeitsbereich Mediensoziologie am Institut für Kommunikationswissenschaft der WWU Münster. 